\title{
Stress entre calouros e veteranos de jornalismo
}

\author{
Journalism freshmen and veterans' stress
}

\author{
Sandra Leal CALAIS \\ Kester CARRARA' \\ Maiara Medeiros BRUM' \\ Karen BATISTA \\ Josiane Keyla YAMADA \\ Juliana Roman Santos OLIVEIRA
}

\section{Resumo}

A vida na universidade corresponde a um período no qual os indivíduos são expostos a influências psicossociais que podem desencadear situações estressoras, prejudicando a saúde e a qualidade de vida dos estudantes. Nessa perspectiva, o objetivo deste estudo foi analisar as diferenças de sintomas, níveis e presença de stress entre calouros $(n=57)$ e veteranos $(n=48)$, de ambos os sexos, do curso de jornalismo de uma universidade pública no interior do Estado de São Paulo. Foi aplicado aos participantes o "Inventário de Sintomas de Stress de Lipp". Dos resultados obtidos, destacam-se: predominância de stress entre as mulheres; maior número de alunos estressados e na fase de quase-exaustão no último ano; predomínio de sintomas físicos entre calouros e psicológicos entre veteranos. Pesquisas são necessárias para elaboração de políticas institucionais que promovam condições favoráveis a um melhor desenvolvimento das potencialidades acadêmicas dos alunos.

Unitermos: qualidade de vida; saúde; stress; universitários.

\begin{abstract}
Universitylife corresponds to a period in which the individuals are exposed to several psychosocial influences that can lead to stressful situations, harming the students' health and quality of life. From this perspective, the objective of the present study was to analyze the symptomatic differences, levels and the presence of stress among freshmen $(n=57)$ and veterans $(n=48)$, of both genders, from a Journalism course of a public university in the countryside of São Paulo State. To the participants, it was delivered the Term of Free Consent, and applied the "Inventory ofSymptoms of Stress for Adults of Lipp". In the obtained results, it was highlighted predominance of stress among women; a bigger number of students stressed and in the phase of barely-exhaustion in the lastyear; predominance of physical symptoms among freshmen and psychological ones among veterans. New researches are necessary to elaborate institutional policies that promote favorable conditions to the students' academic potentialities development.
\end{abstract}

Uniterms: quality of life; health; stress; college students.

O stress, um dos fenômenos que têm afetado as interações sociais, vem sendo discutido em diversas instâncias: no meio acadêmico-científico, nos veículos de comunicação de massa, e nas diversas áreas do conhecimento, como Psicologia, Educação e Saúde. Durante o período de 1984 a 2004 foram publicados no

$\operatorname{cov}$

1 Universidade Estadual Paulista, Faculdade de Ciências, Departamento de Psicologia. Av. Luiz Edmundo Carrijo Coube, 14-01, Vargem Limpa, 17033-360, Bauru, SP, Brasil. Correspondência para/Correspondence to: S.L. CALAIS. E-mail: <scalais@fc.unesp.br>. 
Brasil 232 trabalhos sobre o stress, mostrando a importância do tema em nossa sociedade (Benzoni et al., 2004).

De acordo com a Organização Mundial de Saúde e o Banco Mundial, uma em cada quatro pessoas do mundo sofre de doenças neuropsiquiátricas; representando os distúrbios relacionados ao stress $40 \%$ dessas doenças. O tema do stress tem atingido tamanha proporção, tanto na comunidade acadêmica como entre leigos, que se criou uma "indústria do stress", que diariamente lança propostas de tratamento sem, no entanto, o mínimo de fundamentação teórica e validação científica (O'Gata, 2004).

Segundo Lipp e Malagris (2004) pode-se citar como principais fontes de informação sobre stress pesquisas dentro de instituições de ensino e em organizações não universitárias, pesquisas de populações clínicas e não clínicas e ações empresariais que envolvam levantamento do índice de stress entre os funcionários. O material produzido pode ser encontrado em anais de congressos e em artigos de revistas científicas, livros, internet e trabalhos de metanálise. A relevância da área é evidente: em 2003 ocorreu o I Congresso Brasileiro de Stress e foi fundada a Associação Brasileira de Stress. No Brasil, o Laboratório de Estudos Psicofisiológicos do Stress, criado em 1993, na PUC-Campinas, tem sido considerado uma iniciativa inovadora em função dos muitos estudos desenvolvidos nessa instituição: artigos, teses, dissertações e livros (Cusatis Neto \& Andrade, 2004).

Resulta relevante, portanto, compreender com maior clareza e buscar especificar criteriosamente o conceito de stress e as variáveis que controlam sua ocorrência. Pode-se definir stress como uma reação do organismo diante de um evento estressor - algo que confunda, amedronte ou excite o indivíduo - que desencadeará efeitos físicos e psicológicos (Lipp, 2001).

O conceito de stress tem origem na Física, sendo entendido como o grau de deformidade que uma estrutura sofre quando é submetida a um determinado esforço. A partir desse conceito Selye (1970) define o stress no homem como um conjunto de reações que o organismo tende a desenvolver ao se deparar com uma nova situação que exige um esforço para a adaptação. Em outras palavras, pode-se dizer que o stress representa uma ruptura no equilíbrio do indivíduo, que diante de uma determinada situação é impelido a mudar, a adaptar-se a uma nova realidade, mesmo que ela tenha sido almejada.

É importante ressaltar que o evento estressor pode estar relacionado a uma contingência prazerosa; não necessariamente o stress deve estar associado a condições aversivas. Nesse sentido, Selye define dois tipos de stress: o eustress e o distress. O stress é uma tensão diante de uma situação de desafio, que pode representar para o indivíduo uma ameaça ou uma possibilidade de conquista. O termo eustress é usado quando diante dessa condição o indivíduo consegue se adaptar de forma positiva, obtendo ganhos que irão melhorar seu desempenho diante dessa e de outras situações. O termo distress corresponde a uma má adaptação do indivíduo, podendo acarretar grandes prejuízos para ele (Selye, 1975).

A literatura indica Selye como precursor dos estudos sobre stress quando pesquisou padrões de excitação fisiológica identificados em animais de laboratório expostos a diversas fontes estressoras, tanto físicas quanto psicológicas: calor, frio, dor, choques leves e restrição. Selye constatou que os padrões fisiológicos apresentados pelos animais eram os mesmos, independentes da fonte de stress a qual haviam sido submetidos. Assim, esse autor denominou as reações ao stress de síndrome da adaptação geral, um modelo de resposta ao stress dividido em três fases: alarme, resistência e exaustão (Selye, 1970). Lipp e Malagris (2001) propõem um modelo quadrifásico, acrescentando ao modelo de Selye a fase de quase-exaustão, entre as fases de resistência e exaustão.

A fase de alerta, ou alarme, representa o ponto inicial no qual o indivíduo é colocado diante do evento estressor. Nesse momento, há a quebra da homeostase visando condições de enfrentamento da situação estressora, verificada através da produção de noradrenalina no sistema nervoso simpático e adrenalina pela medula da supra-renal (Lipp, 2001).

Quando o indivíduo é exposto a um estressor de longa duração, ou de grande intensidade, o organismo tende a restabelecer a homeostase rompida na fase anterior e entra na fase de resistência. Isso requer um grande gasto de energia, o que muitas vezes pode levar à sensação de desgaste que será proporcional à quantidade de esforço empregado em reequilibrar-se. Se o organismo é capaz de se readaptar plenamente, o 
processo do stress é interrompido. Entretanto, quando isso não é possível, o organismo evolui para a fase de quase-exaustão (Lipp, 2004).

A fase de quase-exaustão compreende um período no qual o indivíduo não consegue resistir às tensões e nem recuperar a homeostase, porém ele ainda não sucumbiu totalmente aos agentes estressores. É uma fase de transição, na qual a pessoa oscilará entre momentos de desprazer e ansiedade e momentos tranqüilos. Nesse estágio, o indivíduo pode apresentar-se bastante vulnerável física e psicologicamente, com surgimento de doenças leves que geralmente não oferecem risco de vida, permitindo-Ihe ainda trabalhar e atuar na sociedade (Lipp, 2004).

Caso haja uma dificuldade significativa na tentativa de manter a homeostase, o indivíduo passará à fase de exaustão. Essa fase é representada pela exaustão física e psicológica podendo gerar doenças graves e até resultar na morte do indivíduo (Lipp, 2004).

A questão do stress ocupacional tem se sobressaído e algumas profissões vêm sendo pesquisadas. A relação entre ocupação e saúde e qualidade de vida tem sido debatida em diversas pesquisas sobre stress envolvendo professores (Rossa, 2003), juízes (Oliveira, 2004), enfermeiros (Stacciarini \& Troccoli, 2001), policiais civis (Silveira et al., 2005), bancários (Palacios, Duarte \& Camara, 2002) e vestibulandos (Calais, Andrade \& Lipp, 2003). Apesar de haver pesquisas tratando desse tema, ainda se faz necessário ampliar a literatura, focando aspectos não só da saúde, como também educacionais e comunitários.

As pesquisas citadas foram realizadas com adultos, entretanto outras faixas etárias também estão sujeitas a agentes estressores, fazendo com que esses indivíduos desenvolvam um processo de stress que pode ser identificado tanto na população infantil (Lucarelli \& Lipp, 1999) quanto em adolescentes (Tricoli, 2002).

A adolescência é um período psicossocial de transição entre a infância, onde há total dependência da família, e a adultez, estágio caracterizado tanto pela independência social como econômica em relação aos pais. $\mathrm{O}$ adolescente nessa fase é exposto a diversas mudanças: desenvolvimento da identidade psicossexual, alterações hormonais, mudanças no comportamento, dismorfismo sexual e desenvolvimento de habilidades intelectuais mais complexas. Esses eventos, muitas vezes, podem se tornar grandes fontes estressoras.

São colocadas diante do jovem várias tarefas e situações até então desconhecidas, tais como busca pela vocação e profissionalização, pela autonomia e estabilidade, por um relacionamento afetivo, pela identidade e pelo papel que esse jovem desempenhará na sociedade.

Partindo-se do pressuposto de que o adolescente e o adulto jovem pertencem a grupos significativamente expostos e vulneráveis às influências psicossociais, é necessário compreender como se manifesta o stress nessa parcela da população. Assim será possível desenvolver programas que trabalhem essa questão com eficiência, bem como um programa de prevenção dos efeitos negativos do stress no ser humano, uma vez que já se fez referência à ligação do stress com a ocorrência de várias doenças, além de sentimentos desagradáveis e comportamentos destrutivos (AaltoSetälä, 2002).

O ingresso na universidade pode colocar os alunos em contato com estressores específicos, tais como medo, ansiedade e inseguranças geradas pelas possíveis dúvidas e desilusões com a carreira escolhida, aquisição de novas e maiores responsabilidades, problemas na moradia, distanciamento da família, dificuldade no estabelecimento de relacionamento afetivo.

Ross, Niebling e Heckert (1999) realizaram um estudo utilizando a escala The Student Stress Survey (SSS) para determinar as maiores fontes de stress entre universitários. Essa pesquisa contou com cem estudantes de ambos os sexos. Os dados encontrados foram distribuídos da seguinte forma: $38 \%$ das fontes de stress relativas ao âmbito intrapessoal, 28\% ao ambiental, 19\% ao interpessoal e 15\% ao nível acadêmico. As respostas que mais apareceram foram relacionadas às complicações diárias. O âmbito intrapessoal foi a fonte mais comum de stress, sendo cinco os principais tipos de estressores: mudança de hábitos alimentares, do sono, novas responsabilidades, carga de trabalho mais pesada e férias e feriados. Dificuldades financeiras e mudanças nas atividades sociais também foram apresentadas como estressores.

Com a intenção de descrever fatores de stress entre estudantes, Polo, Hernández e Pozo (1996) realizaram um estudo com 64 universitários do curso 
de Psicologia da Universidade Autônoma de Madri. Era apresentada aos estudantes uma lista com onze situações potencialmente geradoras de stress em um âmbito acadêmico. A cada situação os estudantes deveriam associar as respostas que eles acreditavam apresentar diante daquelas experiências estressoras coração disparado, medo, insegurança, vontade de fumar, entre outras - a uma escala de um a cinco para maior ou menor intensidade da resposta. Entre as situações de maior intensidade de respostas de stress citadas pelos alunos, destacaram-se:" falta de tempo para cumprir as atividades acadêmicas", "sobrecarga acadêmica", "realização de uma prova", "exposição de trabalhos em classe", "realização de trabalhos obrigatórios para nota", "tarefas de estudo"e "intervenção em aula"(responder a uma pergunta do professor ou realizar perguntas).

Polo et al. (1996) e Gregorio, Rodríguez, Borda e Río (2003) realizaram uma investigação que visava analisar a influência e a relação existente entre stress e rendimento acadêmico de estudantes universitários. Entretanto os resultados não demonstraram haver correlações significativas entre o desempenho e o nível de stress vivenciado pelos graduandos, e sim relacionaram as qualificações acadêmicas dos estudantes com outras variáveis, tais como padrões de conduta e idade.

Misra e Mckean (2000) analisaram, dentre outros aspectos, o stress em estudantes de uma universidade do centro-oeste americano. Nessa pesquisa, os estudantes mais novos, de primeiro e segundo ano, foram os que apresentaram maior nível de stress.

Em um estudo longitudinal com 308 calouros, Wei, Russel e Zakalik (2005) analisaram a auto-eficácia social, sentimentos de solidão e depressão, chamando a atenção para a adaptação de estudantes que iniciam a vida universitária.

O último ano da graduação também pode apresentar situações estressoras, como trabalhos de conclusão de curso e pressões do mercado de trabalho. Dessa maneira, é relevante para os profissionais que atuam com adolescentes e adultos jovens conhecer quais as manifestações do stress no jovem, quais queixas são esperadas para calouros e veteranos e quais fatores poderiam ser determinantes ou influenciar no nível de stress.

Buscando-se comparar a exposição a estressores 72 acadêmicos, bem como as formas de reação a esses estressores entre estudantes universitários norte-americanos e de outras nacionalidades, Misra e Castillo (2004) realizaram uma pesquisa em duas universidades dos Estados Unidos com alunos cuja idade média era 21 anos entre os americanos, e 25 entre os demais. Consideraram como estressores acadêmicos básicos presentes na vida universitária: a pressão familiar, as exigências do curso, a competição com colegas, o apoio financeiro e o denso conteúdo a ser assimilado em pouco tempo. Os estudantes estrangeiros, além desses fatores, sofriam também com a falta de comunicação com suas famílias (e possível privação de suporte afetivo), as dificuldades de adaptação a uma nova cultura e as limitações de financiamento de seus estudos, uma vez que esses benefícios são destinados prioritariamente aos estudantes nativos.

Contrariando a própria hipótese, os dados de Misra e Castillo (2004) mostraram que os estudantes norte-americanos percebiam um maior stress acadêmico que os estudantes estrangeiros. Os norte-americanos demonstraram como maior fonte de stress acadêmico a autocobrança, que pode ser atribuída tanto ao fato de terem crescido num país onde competir e vencer é valorizado, quanto à hipótese de eles terem sido incentivados a relatar seus sentimentos e assim reconhecer estressores.

Chapell et al. (2005), pesquisando 4 mil estudantes quanto ao desempenho acadêmico e ansiedade, identificaram que as mulheres não só apresentavam melhor desempenho do que os homens, mas também maior ansiedade.

Calais et al. (2003) também indicaram em seu estudo a diferença de sexo na manifestação de stress, apontando o sexo feminino como o que apresenta maior incidência de sintomas, mesmo considerando os aspectos socioculturais.

Estudos sobre o tema são importantes para o desenvolvimento de programas que promovam o controle do stress, tanto para prevenir quanto para tratar daqueles que já se apresentam estressados.

Nesse contexto, foi objetivo do presente estudo comparar os níveis, os sintomas e a presença de stress em universitários calouros e veteranos, de modo a investigar a associação do stress com escolaridade e sexo. 


\section{Método}

\section{Participantes}

Participaram da pesquisa 105 alunos, sendo 57 do primeiro ano do curso de Jornalismo, com idade variando entre 18 e 24 anos, 36 mulheres e 21 homens, e 48 alunos último-anistas (quarto ano) também de Jornalismo, com idade entre 21 e 26 anos, com 35 mulheres e 13 homens, estudantes de uma universidade pública do interior do Estado de São Paulo. A escolha pelo curso de Jornalismo foi por conveniência, além de ser um curso no qual o número de homens e de mulheres é mais equilibrado do que o de outros da mesma universidade.

\section{Material}

Na avaliação do stress foi utilizado o Inventário de Sintomas de Stress de Lipp (ISSL) (2000), constituído de uma lista de sintomas físicos - nó no estômago, sensação de desgaste físico constante, insônia - e psicológicos - entusiasmo súbito, irritabilidade excessiva, hipersensibilidade emotiva - divididos em três quadros, correspondendo cada quadro a uma das fases do modelo. O respondente deve indicar primeiramente quais sintomas do primeiro quadro experimentou nas últimas 24 horas. A seguir, deve assinalar que sintomas sentiu na última semana dentre os apresentados no quadro 2 e, finalmente, deve assinalar, dentre os sintomas físicos e psicológicos do quadro 3, quais apresentou no último mês. O ISSL permite diagnosticar se a pessoa tem stress, em que fase do processo se encontra (alerta, resistência, quase-exaustão e exaustão) e se sua sintomatologia é mais típica da dimensão física ou psicológica.

Foi anexada ao ISSL uma ficha de identificação que solicitava os seguintes dados básicos dos participantes: iniciais dos nomes, sexo, data de nascimento, religião, profissão, situação amorosa, situação de moradia, e, para aqueles que não moravam com a família, freqüência de visita à família.

\section{Procedimentos}

A pesquisa foi realizada em conformidade com a resolução MS 196/96 do Conselho Nacional de Saúde e a Resolução CFP no 016/2000 de 20 de Dezembro de 2000, que trata da ética em pesquisas com seres humanos e deliberação do Comitê de Ética em Pesquisa da Faculdade onde foi realizado o estudo e registro no Sisnep - FR - 105787. Todos os participantes colaboraram por livre vontade e subscreveram o Termo de Consentimento Livre e Esclarecido.

A coleta foi feita durante o período letivo, próxima ao fim do primeiro semestre, sendo o inventário aplicado no início ou término da aula, conforme permissão do professor presente na sala. Foi dada uma explicação prévia de como responder ao instrumento, não havendo tempo estipulado para o seu preenchimento.

Foram verificadas as relações entre presença de stress e escolaridade, presença de stress e sexo, nível de stress e escolaridade $(\mathrm{gl}=1)$ e prevalência de sintomas e escolaridade $(\mathrm{gl}=2)$ através do teste de qui-quadrado $\left(\chi^{2}\right)$, adotando-se uma significância de 5\%.

\section{Resultados}

A análise da ficha de identificação não indicou diferença estatisticamente significativa nas informações sociodemográficas entre os respondentes com e sem stress, exceto pelo sexo.

A amostra pode ser caracterizada em sua maioria por estudantes do sexo feminino (65\%), de religião católica (43\%), solteiros, o que inclui não ter namorado(a) (70\%), moradores de república (61\%) e que visitam suas famílias com uma freqüência quinzenal (32\%).

Os estudantes foram identificados como apresentando ou não stress, segundo os critérios estabelecidos no Inventário de Sintomas de Stress de Lipp. Dos 105 participantes da pesquisa, 54\% apresentavam stress; sendo desse total, $51 \%$ da amostra de alunos do primeiro ano e 60\% de quarto-anistas. De acordo com a análise estatística $\left(\chi^{2}=1,339\right)$, esses números não são suficientes para estabelecer uma relação significativa entre ano escolar e presença de stress.

Diferenças também foram notadas entre as quantidades de homens e mulheres avaliados como tendo stress. Entre as mulheres, 56\% o apresentavam, porém, entre os homens, essa taxa caiu para menos da metade: apenas 27\% apresentavam stress. Estatistica- 
mente, os dados $\left(\boldsymbol{\chi}^{2}=13,882\right)$ corroboram essa relação, mostrando que a variável sexo exerce influência na presença do stress, estando as mulheres mais suscetíveis a ele.

No primeiro ano, 61\% das mulheres tinham stress contra 29\% dos homens. Entre os alunos do quarto ano a diferença ainda é maior: são 75\% de mulheres com stress para 31\% dos homens.

Os alunos estressados se enquadraram em apenas duas das quatro fases relatadas no ISSL: resistência e quase-exaustão. Entre os calouros apenas um aluno estava na fase de quase-exaustão, já no grupo dos veteranos cinco encontravam-se nessa fase. Apesar de haver um número maior de veteranos nessa fase, estatisticamente os números obtidos $\left(\chi^{2}=2,725\right)$ também não são relevantes para sugerir que alunos que se encontram no último ano sejam mais propensos a maiores níveis de stress.

Em relação à sintomatologia, 14 alunos do primeiro ano em fase de resistência tiveram predominância de sintomas físicos, enquanto o calouro em quase-exaustão apresentou uma freqüência igual de tipos de sintomas. No quarto ano, dos 24 alunos em fase de resistência, seis expressaram prevalência de sintomas físicos e 13, de psicológicos. Os cinco estudantes em fase de quase-exaustão apresentaram predominância de sintomas psicológicos, mas o teste do qui-quadrado não aponta nenhuma relação significativa nesses dados $\left(\chi^{2}=6,182\right)$.

Nos dados da ficha de identificação não houve diferenças expressivas entre os alunos com e sem stress. Houve pequena diferença para as respostas de tipo de moradia e visita à família. A maioria dos estudantes morava em repúblicas, 57\% no primeiro ano e 64\% no quarto ano. Para os calouros, a quantidade de alunos com e sem stress moradores em república é quase a mesma, 17 sem stress e 16 com stress. Os dados dos alunos veteranos mostram 13 alunos sem stress contra 18 com stress, para o mesmo tipo de moradia.

Diferenças também podem ser observadas em relação à freqüência de visita à família. Quando a visita é feita quinzenalmente, notam-se nove estudantes sem stress e cinco com stress para os alunos do primeiro ano. Para os alunos quarto-anistas, em relação ao mesmo intervalo de tempo, têm-se três alunos sem stress e 13 74 comstress.

\section{Discussão}

Independente da idade ou área de atuação, as mulheres normalmente apresentam os maiores níveis de stress, o que pode ser notado na pesquisa de Lipp e Tanganelli (2002), na qual foram analisados os níveis de stress e qualidade de vida em magistrados da justiça do trabalho. Esse fato também pode ser confirmado pela pesquisa de Calais et al. (2003), e pela pesquisa de Misra e McKean (2000), na qual se verificou a prevalência de stress em mulheres.

Misra e Castillo (2004) também obtiveram nos resultados de sua pesquisa que tanto as estudantes universitárias norte-americanas quanto aquelas de outras nacionalidades apresentaram mais reações ao stress do que os estudantes do sexo masculino.

Misra, Crist e Burant (2003) investigaram as relações presentes no stress cotidiano, suporte social, estressores acadêmicos e reações a estressores em universitários estrangeiros que estudam nos Estados Unidos, e de acordo com seus resultados não houve diferenças significativas entre os sexos quanto aos estressores cotidianos. Entretanto, em relação ao tipo de reação ao stress (psicológica, comportamental, cognitiva e emocional), foram verificadas diferenças bastante expressivas, comparando-se os estudantes de ambos os sexos.

Na presente pesquisa, esses dados também podem ser observados, ou seja, entre os calouros e veteranos do curso de Jornalismo analisados, o número de mulheres com stress foi maior do que o de homens, conforme diferença significativa apontada através do teste de qui-quadrado disposto nos resultados.

A prevalência de sintomas físicos nos alunos do primeiro ano, contrapondo-se à predominância de sintomas psicológicos presentes nos quarto-anistas, pode ser respaldada com informações encontradas na literatura mundial, como a revisão bibliográfica realizada por Mori (2000), que aponta que os estudantes americanos tendem a manifestar reações emocionais ao stress, ao contrário dos estudantes estrangeiros que manifestam, na maioria, reações físicas.

Esses dados sugerem que os veteranos brasileiros podem estar expostos a variáveis semelhantes aos estudantes americanos e, portanto, desenvolvem a mesma tipologia sintomática. Da mesma forma, os 
calouros brasileiros e os estudantes estrangeiros, ambos inseridos num contexto marcado pela necessidade de adaptação a uma situação nova, apresentariam uma tendência semelhante quanto ao tipo de reação ao stress, a psicológica. No entanto essa variável precisaria ser melhor analisada.

A mudança das solicitações do ambiente acadêmico aos estudantes já no início de seus estudos universitários pode gerar um aumento de responsabilidade, ansiedade e competição bem como possíveis incertezas em relação à escolha profissional, o que pode tornar o contexto universitário um ambiente que favoreça a condição de stress.

Os dados da presente pesquisa, embora não exibam a mesma significância estatística dos resultados de Calais et al. (2003), que relatam que os alunos do quarto ano do ensino superior do curso de Odontologia em uma escola particular apresentaram uma prevalência de stress em relação aos alunos do primeiro ano, evidenciam resultados com tendência semelhante. Neste trabalho houve uma ligeira predominância de alunos com stress no quarto ano, sugerindo assim que haja outras variáveis, como curso, cidade, instituição pública ou privada e tamanho da amostra, que poderiam ser analisadas em novos estudos.

Pode-se atribuir o menor número de alunos com stress no primeiro ano a fatores como aumento de responsabilidade, ansiedade e competição, que podem ser equilibrados pelas situações prazerosas também proporcionadas pelo ingresso na vida acadêmica, tais como liberdade, autonomia, aquisição de novas experiências e desenvolvimento de habilidades sociais. Essa situação já não é novidade para os alunos do quarto ano e variáveis como a rotina, a convivência das repúblicas e maiores responsabilidades podem sobrepor-se a elas.

Porém esses dados diferem daqueles obtidos por Misra e McKean (2000) que apontavam os anos iniciais como mais estressores. Segundo os mesmos autores, os estudantes mais velhos conseguem organizar de forma mais efetiva seus compromissos acadêmicos, apresentando assim conseqüentemente menos stress e ansiedade. Há que se considerar que os contextos universitário e social diferem de um país para outro.

Há um dado sugestivo quanto à moradia dos estudantes. Durante o primeiro ano as pessoas que "montam a república" ainda não se conhecem totalmente, as relações ainda são de descobertas e não há grandes conflitos. Ao chegar ao quarto ano, supondo que a república ainda seja formada pelos mesmos moradores, eles já estão mais íntimos. Defeitos, modos e costumes não antes revelados são agora conhecidos entre a maioria, o que poderia ocasionar desordens e intrigas. Tais dificuldades poderiam ser fatores influentes no aparecimento da situação de stress. Essa condição poderia ser mais bem revista em outro estudo.

Interessante também o dado sobre as visitas às famílias, que levanta a questão do suporte familiar no princípio e no final do curso. Neste estudo, os formandos que visitam suas famílias quinzenalmente apresentaram maior freqüência de stress, sendo aventadas algumas hipóteses quanto à condição estressora: as horas perdidas em viagem impediriam a dedicação necessária aos estudos, e a dificuldade de readaptação ao estilo de vida da família uma vez que esses estudantes aprenderam novas formas de comportamento. Por outro lado, o maior nível de stress os levaria a buscar o aconchego familiar, aumentando o número de visitas à família. Em virtude do número de variáveis, outras pesquisas são necessárias.

\section{Considerações Finais}

Pesquisas vêm demonstrando que os estudantes universitários têm apresentado níveis de stress no decorrer de suas vidas acadêmicas pelo fato de se depararem com mudanças que podem ser consideradas fontes estressoras, como, por exemplo, o aparecimento de novas responsabilidades.

No primeiro ano, quando o calouro ingressa na faculdade, há uma série de expectativas, não só em relação ao curso, mas também à vida universitária em geral. Tendo sido a pesquisa realizada em uma universidade pública, há uma grande quantidade de estudantes vindos de diversas regiões, o que os leva a deixar de morar com a família, gerando a necessidade de criar novas habilidades, como aprender a cozinhar, limpar a casa, pagar as contas e fazer compras.

Além disso, os estudantes têm que conciliar essas tarefas com a vida acadêmica (provas, trabalhos) e a vida social (festas, reuniões com os amigos). Tudo 
isso sem o aconchego e suporte familiar. No entanto esses fatores que poderiam ser desencadeadores de stress são contrabalançados pelas situações prazerosas também proporcionadas pela vida acadêmica, tais como liberdade, autonomia, aquisição de novas experiências e desenvolvimento de habilidades sociais.

Entre os alunos do quarto ano, as situações antes prazerosas talvez não estejam mais em evidência: o que era novidade pode ter se transformado em rotina, além das diversas responsabilidades que vão se acentuando com o decorrer dos anos.

O dado estatístico de maior relevância encontrado neste trabalho foi a presença de stress dominante entre as mulheres, corroborando outros trabalhos e alertando para uma condição que não vem sendo cuidada pela saúde pública com programas especiais tanto de orientação quanto de prevenção.

Este estudo foi possível notar além das diferenças em incidência de stress relacionado ao momento acadêmico dos participantes, diferentes variáveis de relativa influência na sua constatação. Dessa forma, novas pesquisas são necessárias de modo a investigar as diferentes fontes estressoras no ambiente acadêmico para que a universidade possa apresentar políticas institucionais que promovam condições para que seus alunos desenvolvam suas potencialidades acadêmicas sem arcar com o custo do stress em sua saúde, garantindo-Ihes, assim, uma melhor qualidade de vida.

\section{Referências}

Aalto-Setälä, T. (2002). Depressive disorders among young adults. Helsinki: Publications of the National Public Health Institute.

Benzoni, P. E., Carneiro, B. V., Ribeiro, D. P. S. A., Peres, M. R., Marchi, R., \& Milaré, S. A. (2004). Tipologia das pesquisas sobre stress no Brasil. In M. E. N. Lipp (Org.), O stress no Brasil: pesquisas avançadas (pp.205-213). Campinas: Papirus.

Calais, S. L., Andrade, L. M. B., \& Lipp, M. E. N. (2003). Diferenças de sexo e escolaridade na manifestação de stress em adultos jovens. Psicologia: Reflexão e Crítica, 16 (2), 257-263.

Chapell, M. S., Blanding, Z. B., Silverstein, M. E., Takahashi, M., Newman, B., Gubi, A., \& McCann, N. (2005). Test anxiety and academic performance in undergraduate and graduate students. Journal of Educational Psychology, 97 (2), 268-274.
Cusatis Neto, R., \& Andrade, V. C. G. (2004). Análise da produção científica de stress da Pontifícia Universidade Católica de Campinas. In M. E. N. Lipp (Org.), O stress no Brasil: pesquisas avançadas (pp.197-204). Campinas: Papirus.

Gregorio, M. A. P. S., Rodríguez, M., Borda, M., \& Río, C. (2003). Estrés y rendimiento académico en estudiantes universitarios. Cuadernos De Medicina Psicosomatica y Psiquiatria de Enlace, (67/68), 26-33.

Lipp, M. E. N., \& Malagris, L. E. N. (2001). O stress emocional e seu tratamento. In B. Rangé (Org.), Psicoterapias cognitivo-comportamentais. Porto Alegre: Artes Médicas.

Lipp, M. E. N., \& Malagris, L. E. N. (2004). O stress no Brasil de hoje. In M. E. N. Lipp (Org.), O stress no Brasil: pesquisas avançadas (pp.215-220). Campinas: Papirus.

Lipp, M. E. N., \& Tanganelli, M. S. (2002). Stress e qualidade de vida em magistrados da Justiça do Trabalho: diferenças entre homens e mulheres. Psicologia: Reflexão e Crítica, 15 (3), 537-548.

Lipp, M. E. N. (2000). Inventário de sintomas de stress para adultos de Lipp - ISSL. São Paulo: Casa do Psicólogo.

Lipp, M. E. N. (2001). Stress: conceitos básicos. In M. E. N. Lipp (Org.), Pesquisas sobrestress no Brasil: saúde, ocupações e grupos de riscos (2a.ed., pp.17-29). Campinas: Papirus.

Lipp, M. E. N. (2004). Stress emocional: esboço da teoria de "temas de vida". In M. E. N. Lipp, (Org.), O stress no Brasil: pesquisas avançadas (pp.17-28). Campinas: Papirus.

Lucarelli, M. D. M., \& Lipp, M. E. N. (1999). Validação do inventário de sintomas de stress infantil - ISS - I. Psicologia: Reflexão e Crítica, 12 (1), 71-88.

Misra, R., \& Castillo, L. G. (2004). Academic stress among college students: comparison of American and International students. International Journal of Stress Management, 11 (2), 132-148.

Misra, R., \& Mckean, M. (2000). College students' academic stress and its relation to their anxiety, time management, and leisure satisfaction. American Journal of Health Studies, $16(1), 41-51$.

Misra, R., Crist, M., \& Burant, C. J. (2003). Relationships among life stress, social support, academic stressors, and reactions to stressors of international students in the United States. International Journal of Stress Management, $10(2), 137-157$.

Mori, S. C. (2000). Addressing the mental health concerns of international students. Journal of Counseling and Development, 78, 137-144.

O'Gata, A. (2004). Prefácio. In M. E. N. Lipp (Org.), O stress no Brasil:pesquisas avançadas (pp.11-13). Campinas: Papirus.

Oliveira, J. B. (2004). Fontes e sintomas de stress em juízes e servidores públicos: diferenças entre homens e mulheres. Dissertação de mestrado em Psicologia Clínica não-publicada, Pontifícia Universidade Católica de Campinas.

Palacios, M., Duarte, F., \& Camara, V. M. (2002). Trabalho e sofrimento psíquico de caixas de agências bancárias na cidade do Rio de Janeiro. Caderno de Saúde Pública, 18 (3), 843-851. 
Polo, A., Hernández, J. M., \& Pozo, C. (1996). Evaluación del estrés académico en estudiantes universitarios. Ansiedad y Estrés, 2 (2-3), 159-172.

Ross, S. E., Niebling, B. C., \& Heckert, T. M. (1999). Sources of stress among college students. College Student Journal, 33 (2), 312-317.

Rossa, E. G. O. (2003). Relação entre o stress e o burnout em professores do ensino fundamental emédio. Dissertação de mestrado em Psicologia não-publicada, Pontifícia Universidade Católica de Campinas.

Selye, H. (1970). The evolution of the stress concept: stress and cardiovascular disease. In L. Levi. Society, stress and disease (Vol.1, pp.299-311). London: Oxford University Press.

Selye, H. (1975). Stress and distress. Comprehensive therapy, 1 (8), 9-13.

Silveira, N. M., Vasconcellos, S. J. L., Cruz, L. P., Kiles, R. F., Silva, T. P., Castilhos, D. G., \& Gauer, G. J. C. (2005).
Avaliação de burnout em uma amostra de policiais civis. Revista de Psiquiatria do Rio Grande do Sul, 27 (2), 159-163.

Stacciarini, J. M. R., \& Troccoli, B. T. (2001). O stress na atividade ocupacional do enfermeiro. Revista Latino-Americana de Enfermagem, 9 (2), 17-25.

Tricoli, V. A. C. (2002). Escala de stress para adolescentes: construção e validação. Tese de doutorado não-publicada, Programa de Pós Graduação em Psicologia, Pontifícia Universidade Católica de Campinas.

Wei, M., Russell, D. W., \&Zakalik, R.A. (2005). Adult attachment, social self-efficacy, self-disclosure, Ioneliness, and subsequent depression for freshman college students: a longitudinal study. Journal of Counseling Psychology, 52 (4), 602-614.

Recebido em: 24/5/2006

Versão final reapresentada em: 1/9/2006

Aprovado em: 14/9/2006 
